\title{
Simulation of Ionic Copolymers by Molecular Dynamics
}

\author{
M. Dzięcielski ${ }^{1}$, P. Knychała ${ }^{2}$, M. Banaszak $^{3 *}$ \\ ${ }^{1}$ Faculty of Geographical and Geological Sciences, Adam Mickiewicz University \\ ul. Krygowskiego 10, 61-680 Poznań, Poland \\ ${ }^{2}$ Faculty of Polytechnic, The President Stanislaw Wojciechowski University School of Applied Sciences in Kalisz \\ ul. Nowy Świat 4, 62-800 Kalisz, Poland \\ ${ }^{3}$ Faculty of Physics, A. Mickiewicz University \\ ul. Umultowska 85, 61-614 Poznan, Poland \\ *E-mail:mbanasz@amu.edu.pl
}

Received: 19 November 2016; revised: 27 November 2016; accepted: 28 November 2016; published online: 09 December 2016

\begin{abstract}
Using GROMACS (a molecular dynamics package) we simulate ionic copolymers and compare the numerical results with those obtained by the lattice Monte Carlo simulations. While the results are qualitatively similar for both methods, the simulation times are significantly longer for the molecular dynamics simulations than those for the corresponding Monte Carlo runs.
\end{abstract}

Key words: GROMACS, molecular dynamics, ion diblock copolymer, microphase separation

\section{INTRODUCTION}

Block copolymers exhibit order-disorder transitions which result in formation of different types of nanostructures such as ordered lamellae, cylinders, spheres or double gyroid phase. In case of non-ionic diblock copolymer melts, one can predict stability windows for different types of morphologies from the Flory interaction parameter, $\chi$, and length of the two blocks, $N_{A}$ and $N_{B}$. The lamellar phase is favored for symmetric chains (when both blocks have similar length), whereas cylinders or spheres are recorded in asymmetric case (when block lengths differ significantly). Such copolymers have been investigated by theory and computer simulations for a long time [1-6]. The agreement between predictions of simulations, self-consistent field theory and experiment is surprisingly good [7].

In recent years, continuing interest in ion-containing block copolymers has also been observed due to their use in clean-energy-related applications such as fuel cells and batteries [8-11]. Microphase separation for ion-containing copolymers is different from that for neutral systems. For example, for symmetric diblock copolymers the non-lamellar nanotructures were observed and lamellar microphase was recorded in the asymmetric case. There have been several efforts to understand the effect of ion-containing monomers on the self-assembly of block copolymers [12-15]. Computer simulations of such copolymers are more demanding because of the long-range interactions which have to be included. Some studies suggest that a minimal lattice model without longrange interactions can be used in the case of ion-containing block copolymers [16-19]. In this model the Flory interaction parameter, $\chi$, between blocks is replaced by an effective interaction parameter that accounts for ionic interactions. This simplification allows us to obtain results which are qualitatively consistent with experimental data.

The aim of this study is to demonstrate that GROMACS, which is a Molecular Dynamics (MD) package, can be applied to simulate ion-containing block copolymers. Specifically, we employ GROMACS to investigate ion-containing copolymers using full electrostatic interactions. This kind of simulations is expected to be much more time consuming compared with the lattice Monte Carlo (MC) simulations 


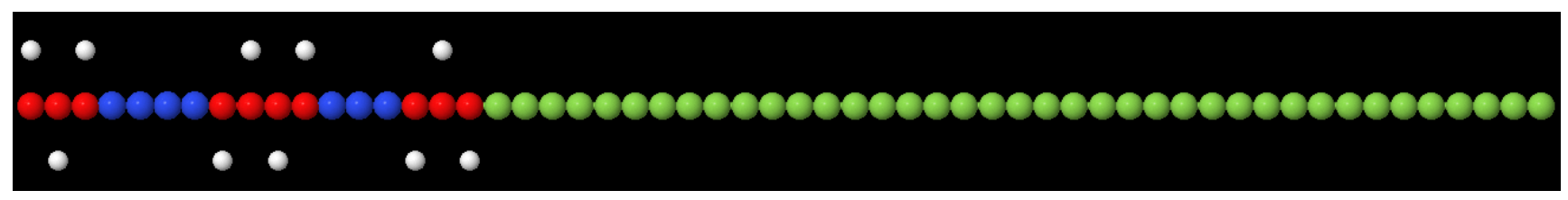

Fig. 1. The coarse-grained model of a chain, used in simulation, consisting of segments $A$ (blue), $S_{+}$(red), $B$ (green), and counterions $S_{-}$(white)

which we performed in our previous publications. In this paper, simulations are limited to a single sequence of segments in a copolymer chain. We chose an asymmetric diblock chain where volume fraction of non-ionic block is 0.7 . This sequence of segments was previously investigated using Monte Carlo simulations in reference [18] and compared with experimental results [11]. The obtained results were surprising because, quite counterintuitively, lamellar nanophase was observed.

\section{MODEL AND METHOD}

The coarse-grained model in which a group of atoms is modeled by a single segment, is widely used in computer simulations of copolymers in order to speed up the calculations. For example, each monomer, $\mathrm{C}_{2} \mathrm{H}_{4}$, consists of 6 atoms. A few such monomers are replaced by a single segment. Such simplification yields results which are consistent with theory and experiment.

The coarse-grained model of poly-(styrenesulfonate)-bpolymethylbutylene (PSS-PMB) is used (Fig. 1). The chain of length $N=56$ consists of three segment's type: $A, S_{+}$, and $B$, which modeled styrene (S), sulfonated styrene (SS), methylbutylene (MB), respectively. The first two types of segments are part of ion-block whose length is 17 . The length of non-ionic block is 39. It means that the chain is asymmetric and volume fraction of non-ionic block is 0.7 . Moreover, the volume fraction of segments $S_{+}$in ionic block, which correspond to experimental sulfonation level, is $p=0.588$. The simulation box contains 59 chains and 590 counterions $S_{-}$. The reduced density is $\rho^{*}=0.5$. The usual periodic boundary conditions are applied. The box is cubic with size equal to $19.8 \sigma$, where $\sigma$ is the size a polymer segment.

The repulsive interactions are modeled via the WCA potential, defined as follows:

$$
U_{W C A}= \begin{cases}4 \varepsilon\left[\left(\frac{\sigma}{r}\right)^{12}-\left(\frac{\sigma}{r}\right)^{6}\right]+\varepsilon & \text { when } r<2^{1 / 6} \sigma \\ 0 & \text { when } r \geq 2^{1 / 6} \sigma\end{cases}
$$

where $\epsilon$ and $\sigma$ are the usual Lennard-Jones parameters [20].

The harmonic potential [21] or finitely extensible nonlinear elastic (FENE) potential [22] is used to modeled bonds between segments. To simulate the system effectively at high temperatures the LINCS [23] algorithm is used, which is the simplest version of SHAKE [24] algorithm.

Moreover, the Coulomb electric potential is applied:

$$
U_{C}=\frac{1}{4 \pi \varepsilon_{0} \varepsilon_{r}} \cdot \frac{Q}{r},
$$

where $\epsilon_{0}$ is the vacuum permittivity, and $\epsilon_{r}$ is the relative permittivity. The system contains neutral segments ( $A$ and $B$ ) as well as ionic elements $S_{+}$and $S_{-}$with value of charges +1 and -1 , respectively. The number of segments $S_{+}$and $S_{-}$is the same so the whole system is neutral. Because of long-range interactions the Particle Mesh Ewald [25] version of Ewald [26-28] summation is applied in simulations.

The reduced dimensionless temperature, $T^{*}$ is defined as follows:

$$
T^{*}=\frac{k_{B} T}{E_{\sigma}},
$$

where $T$ is the absolute temperature, $k_{B}$ is the Boltzmann constant and

$$
E_{\sigma}=\frac{1}{4 \pi \varepsilon_{0}} \cdot \frac{e^{2}}{\sigma}
$$

Initially the polymer chains assume statistical conformations, random orientations, and are uniformly distributed within the simulation box. Next, we run thermal simulation, starting with initial velocities of segments, which are proportional to the square root of temperature. The NVT thermostat by Nose and Hoover [29, 30] is used to ensure adequate temperature. Equations of motion are solved using a leapfrog algorithm [31], a variety of the Verlet [32] method. The time step used in simulations is optimized for performance. The simulations are repeated at least 3 times starting from different initial states.

\section{RESULTS AND DISCUSSION}

The simulations were performed for 15 different value of temperature, $T^{*}$, which are expressed in reduced units [33]. 
a)

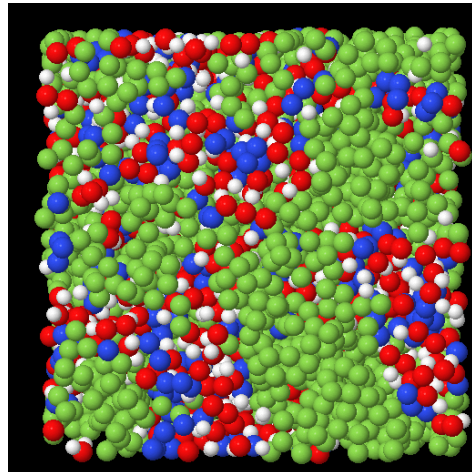

b)

c)

d)

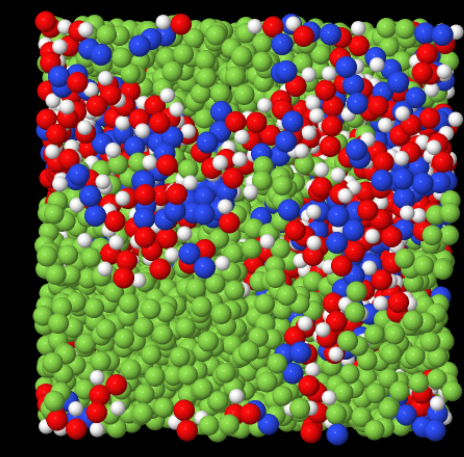

\section{)}

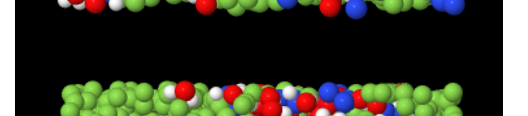

\section{(1)}
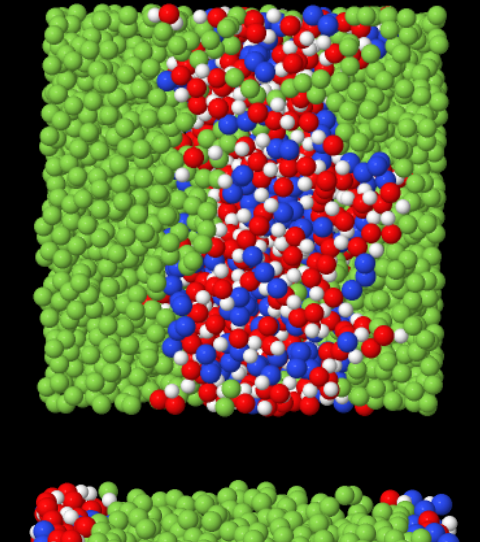

Q.

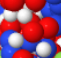

is

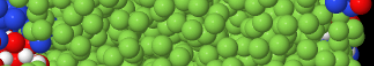
. 013935350 108335353535 8.0353535350 - 15350 1.233535 8. 8. 800000000

Fig. 2. Selected nanostructures from simulations: a) disordered phase at $T^{*}=0.18 \mathrm{~b}$ ) disordered phase at $T^{*}=0.12$, c) lamellar phase at $\left.T^{*}=0.084, \mathrm{~d}\right)$ lamellar phase at $T^{*}=0.042$. The segments $A$ are shown in blue, $S_{+}$in red, $B$ in green, and counterions $S_{-}$in white
Typical snapshots from simulations are shown in Fig. 2. In particular, at high temperatures $\left(T^{*}=0.18\right.$ and 0.12$)$ the disordered nanophase is observed (Fig. 2 a, b), and at low temperatures $\left(T^{*}=0.084\right.$ and $\left.T^{*}=0.042\right)$ the lamellar nanostructure is obtained (Fig. $2 \mathrm{c}$ and d). The structure factor is calculated using the following equation:

$$
\begin{aligned}
S(\vec{k})=\frac{1}{n_{\alpha}} & \left\langle\left(\sum_{m=1}^{n_{\alpha}} \cos \left(\vec{k} \cdot \vec{r}_{m}\right)\right)^{2}\right. \\
& \left.+\left(\sum_{m=1}^{n_{\alpha}} \sin \left(\vec{k} \cdot \vec{r}_{m}\right)\right)^{2}\right\rangle_{\text {thermal average }}
\end{aligned}
$$

The simulated structure factors are presented in Fig. 3. At $T^{*}=0.18$ and 0.12 , we can observe a single broad peak which is characteristic for a nanostructure without long range arrangement. The $S(k)$ calculated for $T^{*}=0.084$ and 0.042 are also presented in Fig. 3, where one can observe the firstorder peak $k^{*}$ as well as the high-order peaks. The ratio $k / k^{*}=1$ and 2 is observed at $T^{*}=0.084$. Additionally, at $T^{*}=0.042$, the third peak for $k / k^{*}=4$ is obtained. The ratios $k / k^{*}$ indicate the lamellar nanophase.

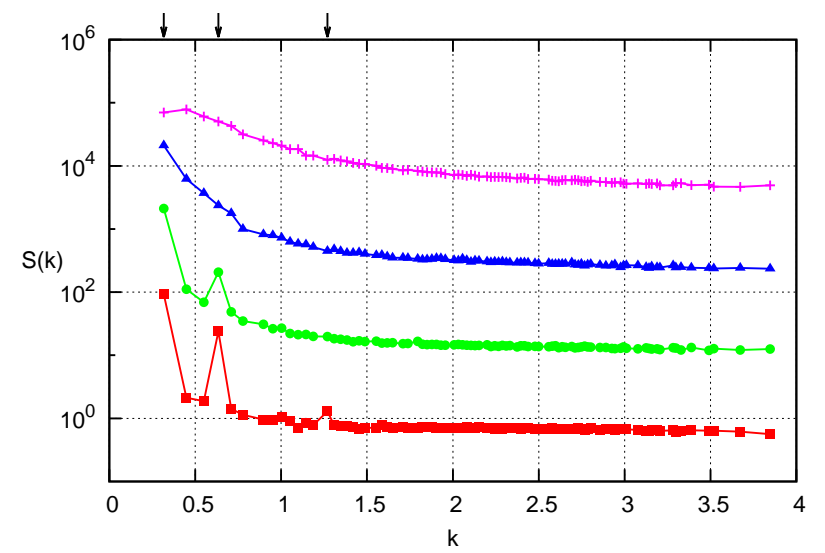

Fig. 3. Structure factor calculated do $\alpha=B$ at: $T^{*}=0.18$ (pink crosses), $T^{*}=0.12$ (blue triangles), $T^{*}=0.084$ (green circles), $T^{*}=0.042$ (red squares). The arrows show peaks at $k / k^{*}=1$, 2 , and 4 indicate $\mathrm{L}$ phase. The function for each temperature are shifted for clarity

The average concentration profiles of $A+S$ (ion blocks) and $B$ (non-ion blocks) are plotted as a function of distance along the vector normal to the layer in Fig. 4. At $T^{*}=0.18$ and 0.12 , the peaks in the profiles are broad, which means that blocks of different types are not fully separated. At lower temperatures $\left(T^{*}=0.084\right.$ and 0.042$)$ the results are completely different because one can observe clear peaks. The interface between ion and non-ion blocks becomes more sharp and clear. 

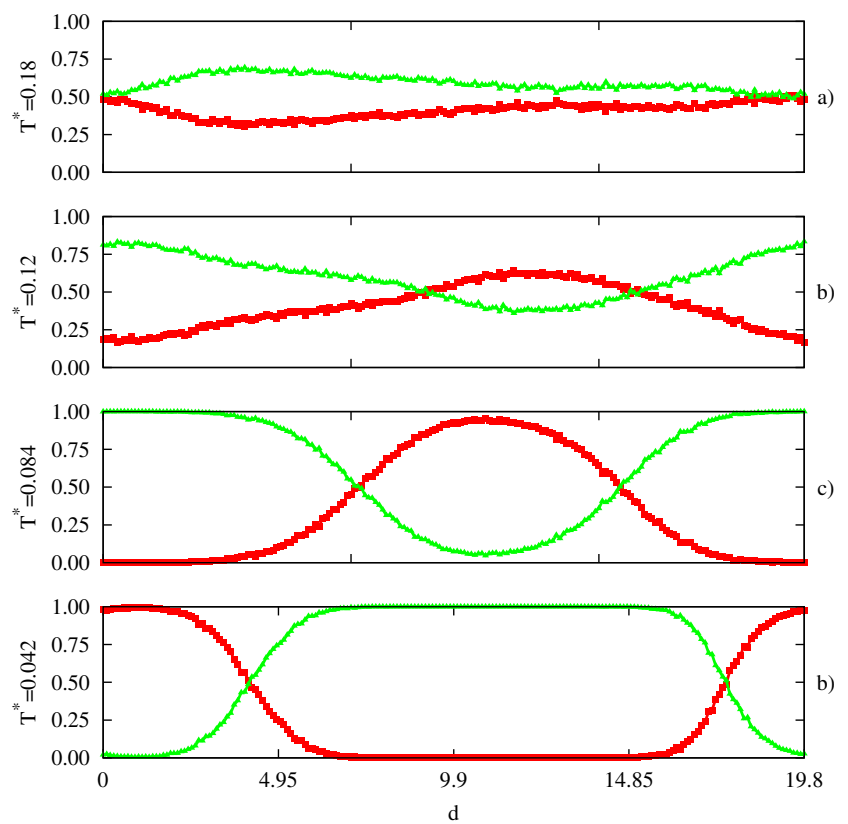

Fig. 4. Monomer concentration profiles at: a) $T^{*}=0.18$ (disordered phase), b) $T^{*}=0.12$ (disordered phase), c) $T^{*}=0.084$ (L phase), a) $T^{*}=0.042$ (L phase). The profiles for $A+S$ and $B$ segments are shown in red and green, respectively

To determine order-disorder transition temperature, $T_{O D T}^{*}$, more precisely, we calculated the structure factor for four selected $T^{*}$ 's near microphase separation temperature. The results are presented in Fig.5. At $T^{*}=0.108$, the $S(k)$ is smooth with one broad peak which means that disordered nanophase is recorded. At $T^{*}=0.102$, an additional peak is observed which, means that the system starts to self-assembly. This second order peak becomes increasingly clear and, at two lower temperatures, the high-order peaks appear. Using these results one can estimate $T_{O D T}^{*}$ to be about 0.099 .

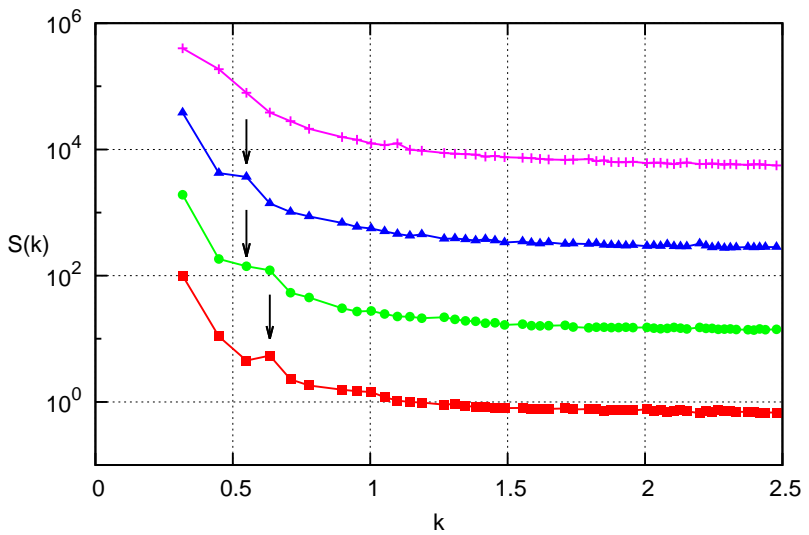

Fig. 5. Structure factor at $T^{*}=0.108$ (pink crosses), $T^{*}=0.102$ (blue triangles), $T^{*}=0.096$ (green circles), $T^{*}=0.090$ (red squares). The function for each temperature are shifted for clarity
It is surprising that for an asymmetric diblock copolymer with volume fraction non-ion block 0.7 , the lamellar nanostructures are observed. The cylindrical nanophase is recorded for non-ionic diblock for the same volume fraction [3, 34]. The same results were obtained experimentally [11] as well as with Monte Carlo simulations, where coarse-grained block copolymers were studied by a minimal lattice model with short-range interactions [18]. Application of such a simplified model to investigate ion-copolymers is not obvious. Those results suggest that both methods allow us to obtain qualitatively consistent results. The MD is a more accurate method but significantly more time consuming. It would take more time to obtain full phase diagrams (as in references [16-18]) depending on different lengths of chain, symmetry and segment sequences using molecular dynamic simulations with long-range interactions.

To compare the efficiency of both methods, we performed simulations of a test system using molecular dynamics (MD) with GROMACS software and the lattice Monte Carlo (MC) method. We recorded the high-order peaks in $S(k)$ to determine when the microphase separation takes place. In case of molecular simulations, it took $2.1 \cdot 10^{6}$ simulations steps. It corresponds to $8.2 \cdot 10^{5}$ real seconds of CPU using. The Monte Carlo simulations took $8 \cdot 10^{5}$ Monte Carlo steps which corresponds to 120 real seconds. The results are summarized in tab. 1. The MD simulation took nearly 7000 more times of CPU time than calculations using the MC method. It is related to a huge number of floating point calculations in $\mathrm{MD}$, and also with taking into account the long range electrostatic interactions.

Tab. 1. Time of simulation of test system using molecular dynamics (GROMACS software) and lattice Monte Carlo calculation

\begin{tabular}{l|c}
\hline Method & time [s] \\
\hline Molecular dynamics & 820800 \\
\hline Monte Carlo & 120 \\
\hline
\end{tabular}

\section{CONCLUSIONS}

The molecular simulations of asymmetric ionic diblock copolymers with long range interactions are performed using GROMACS. Simulations have been limited to a single sequence of segments. The lamellar nanostructure is observed for asymmetric diblock copolymers where volume fraction of non-ionic block is 0.7 . It is a surprising result since all copolymer theories predict non-lamellar phases for non-ionic diblock copolymers at this volume fraction. Nevertheless, this result is qualitatively consistent with that obtained for a minimal lattice model of Monte Carlo simulations. Moreover, the result is in accordance with the experimental result of PSS-b-PMB. The simulations also show that the MD method is significantly more time consuming that the lattice MC. This result suggests that using a simple lattice model to simulate 
ion-containing melts, like PSS-b-PMB, is appropriate, which was not obvious previously.

\section{Acknowledgments}

PK and MB gratefully acknowledge the computational grant from the Supercomputing and Networking Center (PCSS) in Poznan, Poland and grant N N204 018938 from Polish Ministry of Science and Higher Education.

\section{APPENDIX}

\section{1. GROMACS calculations}

GROMACS is one of the most popular software packages designed for simulating, biomolecular systems such as proteins and lipids using a molecular dynamics method [35]. It can also be used to simulate copolymers using coarsegrained model. In case of a huge system, like a copolymers system which contains a lot of chains, calculations are usually made at high performance computing clusters. This software does not have a graphical user interface and the simulations are configured and run using a console text mode. At least three files have to be prepared to start simulation of block copolymers (grompp.mdp, topol.top i conf.gro). Those files are described briefly herein.

The first file, grompp.mdp, contains the following simulation parameters:

- integrator - algorithm used to solve equations of motion, option $m d$ means Verlet algorithm at leap-frog version,

- nsteps - number of simulation steps,

- $n s t l i s t$ - the frequency (in steps of simulation) of updating the list of neighborhood,

- nstxout - the frequency (in steps of simulation) of saving segments coordinates to topol.tpr file, which can be used to calculate structural parameters of investigated system (e.g structure factor),

- nstlog - the frequency (in steps of simulation) of saving information about simulation to $m d . \log$ file,

- $d t$ - time step (in $p s$ ) used to solve equations of motion,

- constraints - information which molecules have bonds,

- ns_type - way of creating the list of neighborhood, option grid means that the grid is created in the simulation box,

- $p b c$ - in which directions the periodic boundary condition are turned on,

- periodic-molecules - turn on/off periodic boundary conditions,

- vdwtype - van der Waals interactions defined by user, in our simulation it is WCA,

- coulombtype - way of long-range interactions calculation, the pme means Particle-Mesh Ewald,
- rlist - maximum distance (in $\mathrm{nm}$ ) used in the list of neighborhood,

- $r v d w$ - van der Waals potential range (in $\mathrm{nm}$ ),

- rcoulomb - electrostatic potential range (in $\mathrm{nm}$ ),

- epsilon- $r$ - the relative dielectric constant,

- tcoupl - thermostat type,

- tc_grps - which segments should be at constant temperature, option system means all segments,

- $t a u \_t$ - the frequency (in steps of simulation) of correction by thermostat,

- nsttcouple - the frequency (in steps of simulation) of coupling the temperature,

- $r e f_{-} t$ - the simulation temperature in Kelvin,

- Pcoupl - the pressure coupling algorithm, option no means no pressure coupling.

GROMACS allows to use interaction potential defined by user [36]. The WCA potential, which is not added by default in this package, is used in our simulations. To use it, the file table.xvg which contains values of selected potential should be created. The details of this procedure are described in GROMACS documentation [37].

The topol.top file contains information about the topological structure of copolymers. It contains several sections which have the following meaning:

- defaults - contains 5 parameters:

- nbfunc - the non-bonded function type (number 1 means Lennard-Jones potential),

- comb-rule - the number of the combination rule (number 2 is used in case of reduced units and Lennard-Jones potential),

- gen-pairs - scaling factor for electrostatic interactions, not used in simulations,

- udgeLJ - the factor by which to multiply LennardJones interactions,

- fudgeQQ - the factor by which to multiply electrostatic interactions,

- atomtypes - information about atoms (name, mass, charge, particle type - value A means atom, sigma and epsilon),

- bondtypes - information about bonds (names atoms in bond, bond type, bond length, bond energy),

- moleculetype - information about name of molecule and number of excluded neighbors for non-bonded interactions.

- atoms - information about atoms (number, type, residue number, residue name, name, charge group number and charge value),

- bonds - information about bonds (numbers of bonded atoms, bond type),

- system - name of our system,

- molecules - name of our molecule.

More details about this file are described in chapter 5.7 of GROMACS documentation [33]. 
The last described file (conf.gro) contains information about initial coordinates of all segments. The used gro format is close to $x y z$ format and can contains the following information:

- title,

- number of segments,

- information about segments,

- the box size.

Each row with information about segments has the following format:

- chain's number (maximum 5 intiger digits) and chains's name (maximum 5 characters),

- segment's name (maximum 5 characters),

- segment's number (maximum 5 intiger digits),

- segment's coordinates (x, y, z; maximum 8 real digits with an accuracy of 3 decimal places),

- segment's velocities (x, y, z; maximum 8 real digits with an accuracy of 4 decimal places).

Those 3 files should be placed in a simulation directory and compiled using grompp command to binary file topol.tpr. The simulation is run using the mdrun command which has many options. One of them is - $n t$ which determines the number of processes to be used in simulation. The $-v$ option allow to see the predicted finish time of calculations.

The final results of simulation are 4 files: confout.gro, $m d . l o g$, ener.edr, and traj.trr. The confout.gro contains the final coordinates of all segments and can be transformed to $x y z$ format, which can be visualized using V-sim, RasmMol or JMol. The energy command can be used to calculate a different type of energy at selected time of simulation. Similarly, the trajconv command can be used to obtain coordinates of segments.

\section{The grompp.mdp file}

\begin{tabular}{|c|c|}
\hline integrator & $=\mathrm{md}$ \\
\hline nsteps & $=1000000$ \\
\hline nstlist & $=10$ \\
\hline nstxout & $=100000$ \\
\hline nstlog & $=50000$ \\
\hline$d t$ & $=0.001$ \\
\hline constraints & $=\mathrm{all}$-bonds \\
\hline ns_type & $=$ grid \\
\hline $\mathrm{pbc}$ & $=x y z$ \\
\hline periodic-molecules & $=$ yes \\
\hline vdwtype & $=$ User \\
\hline coulombtype & $=$ pme \\
\hline rlist & $=1.25$ \\
\hline rvdw & $=1.13$ \\
\hline rcoulomb & $=1.25$ \\
\hline epsilon-r & $=1$ \\
\hline tcoupl & $=$ nose-hoover \\
\hline tc_grps & $=$ system \\
\hline tau_t & $=1$ \\
\hline nsttcouple & $=1$ \\
\hline$r e f \_t$ & $=400$ \\
\hline Pcoupl & $=\mathrm{no}$ \\
\hline
\end{tabular}

\section{The topol.top file}

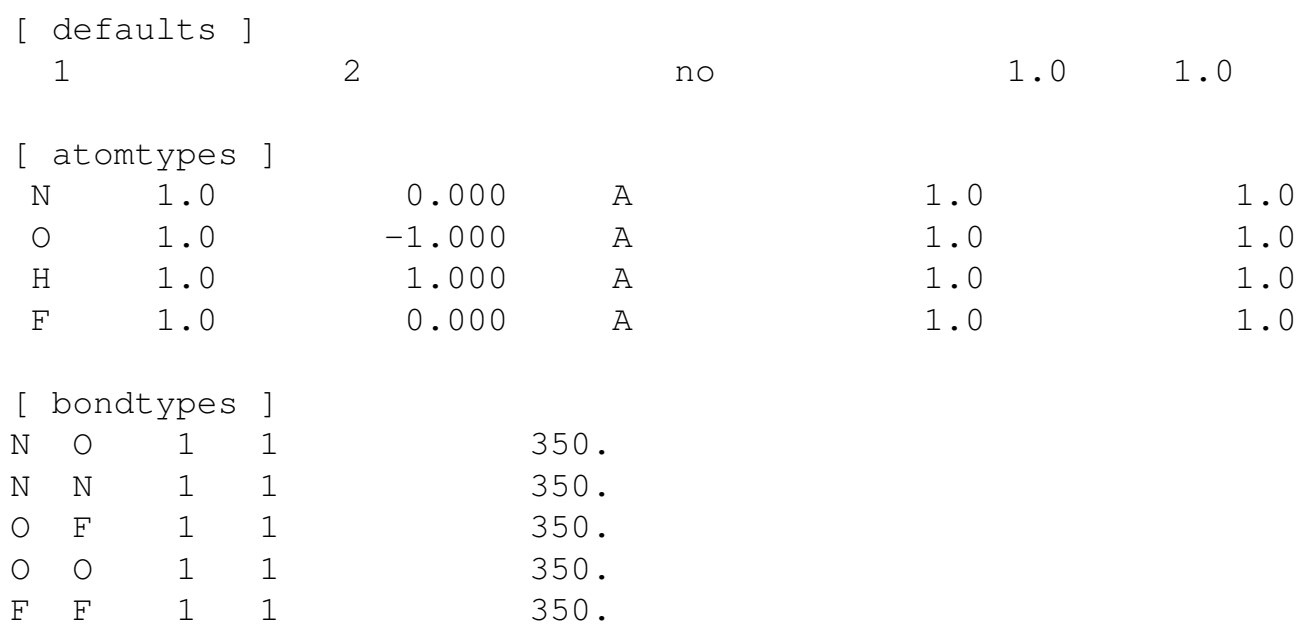




\begin{tabular}{|c|c|c|c|c|c|c|}
\hline \multicolumn{7}{|c|}{ [atoms ] } \\
\hline $\mathrm{nr}$ & type & resnr & residu & atom & cgnr & charge \\
\hline 1 & 0 & 1 & 0 & 01 & 1 & -1.0 \\
\hline 2 & 0 & 1 & 0 & $\mathrm{O} 2$ & 2 & -1.0 \\
\hline 3 & 0 & 1 & 0 & 03 & 3 & -1.0 \\
\hline 4 & $\mathrm{~N}$ & 1 & $\mathrm{~N}$ & N1 & 4 & 0.0 \\
\hline 5 & $\mathrm{~N}$ & 1 & $\mathrm{~N}$ & N2 & 5 & 0.0 \\
\hline 6 & $\mathrm{~N}$ & 1 & $\mathrm{~N}$ & N3 & 6 & 0.0 \\
\hline 7 & $\mathrm{~N}$ & 1 & $\mathrm{~N}$ & N4 & 7 & 0.0 \\
\hline 8 & 0 & 1 & 0 & 04 & 8 & -1.0 \\
\hline 9 & 0 & 1 & 0 & 05 & 9 & -1.0 \\
\hline 10 & 0 & 1 & 0 & 06 & 10 & -1.0 \\
\hline 11 & 0 & 1 & 0 & 07 & 11 & -1.0 \\
\hline 12 & $\mathrm{~N}$ & 1 & $\mathrm{~N}$ & N5 & 12 & 0.0 \\
\hline 13 & $\mathrm{~N}$ & 1 & $\mathrm{~N}$ & N6 & 13 & 0.0 \\
\hline 14 & $\mathrm{~N}$ & 1 & $\mathrm{~N}$ & N7 & 14 & 0.0 \\
\hline 15 & 0 & 1 & 0 & 08 & 15 & -1.0 \\
\hline 16 & 0 & 1 & 0 & 09 & 16 & -1.0 \\
\hline 17 & 0 & 1 & 0 & 010 & 17 & -1.0 \\
\hline 18 & $\mathrm{~F}$ & 1 & F & F1 & 18 & 0.0 \\
\hline 19 & F & 1 & F & F2 & 19 & 0.0 \\
\hline 20 & F & 1 & F & F3 & 20 & 0.0 \\
\hline 3893 & $\mathrm{H}$ & 1 & $\mathrm{H}$ & H58 9 & 3893 & 1.0 \\
\hline 3894 & $\mathrm{H}$ & 1 & $\mathrm{H}$ & H590 & 3894 & 1.0 \\
\hline
\end{tabular}

[bonds ]

\begin{tabular}{|c|c|c|}
\hline ; ai & $a j$ & funct \\
\hline 1 & 2 & 1 \\
\hline 2 & 3 & 1 \\
\hline 3 & 4 & 1 \\
\hline 4 & 5 & 1 \\
\hline 5 & 6 & 1 \\
\hline 6 & 7 & 1 \\
\hline 7 & 8 & 1 \\
\hline 8 & 9 & 1 \\
\hline 9 & 10 & 1 \\
\hline 10 & 11 & 1 \\
\hline 3299 & 3300 & 1 \\
\hline 3300 & 3301 & 1 \\
\hline 3301 & 3302 & 1 \\
\hline 3302 & 3303 & 1 \\
\hline 3303 & 3304 & 1 \\
\hline \multicolumn{3}{|c|}{ [system] } \\
\hline \multicolumn{3}{|c|}{ [molecules] } \\
\hline $\mathrm{PE} 6000$ & & 1 \\
\hline
\end{tabular}

\section{The conf.gro file}

pol 


\begin{tabular}{|c|c|c|c|c|c|c|c|c|}
\hline & & & & & & & & \\
\hline $1 \mathrm{AA}$ & $\mathrm{O} 1$ & 1 & 1.414 & 9.192 & 19.092 & 0.0000 & 0.0000 & 0.0000 \\
\hline $1 \mathrm{AA}$ & $\mathrm{O} 2$ & 2 & 0.707 & 9.192 & 18.385 & 0.0000 & 0.0000 & 0.0000 \\
\hline $1 \mathrm{AA}$ & 03 & 3 & 0.707 & 8.485 & 19.092 & 0.0000 & 0.0000 & 0.0000 \\
\hline $1 \mathrm{AA}$ & N1 & 4 & 0.000 & 7.778 & 19.092 & 0.0000 & 0.0000 & 0.0000 \\
\hline $1 \mathrm{AA}$ & N2 & 5 & 19.092 & 7.778 & 0.000 & 0.0000 & 0.0000 & 0.0000 \\
\hline $1 \mathrm{AA}$ & N3 & 6 & 18.385 & 7.071 & 0.000 & 0.0000 & 0.0000 & 0.0000 \\
\hline $1 \mathrm{AA}$ & N4 & 7 & 18.385 & 7.778 & 19.092 & 0.0000 & 0.0000 & 0.0000 \\
\hline $1 \mathrm{AA}$ & 04 & 8 & 18.385 & 7.071 & 18.385 & 0.0000 & 0.0000 & 0.0000 \\
\hline $1 \mathrm{AA}$ & 05 & 9 & 19.092 & 7.071 & 17.678 & 0.0000 & 0.0000 & 0.0000 \\
\hline $1 \mathrm{AA}$ & 06 & 10 & 0.000 & 7.778 & 17.678 & 0.0000 & 0.0000 & 0.0000 \\
\hline $1 \mathrm{AA}$ & 07 & 11 & 0.707 & 8.485 & 17.678 & 0.0000 & 0.0000 & 0.0000 \\
\hline $1 \mathrm{AA}$ & N5 & 12 & 0.707 & 7.778 & 16.971 & 0.0000 & 0.0000 & 0.0000 \\
\hline $1 \mathrm{AA}$ & N6 & 13 & 0.707 & 7.071 & 17.678 & 0.0000 & 0.0000 & 0.0000 \\
\hline $1 \mathrm{AA}$ & N7 & 14 & 0.000 & 7.071 & 18.385 & 0.0000 & 0.0000 & 0.0000 \\
\hline $1 \mathrm{AA}$ & 08 & 15 & 0.707 & 6.364 & 18.385 & 0.0000 & 0.0000 & 0.0000 \\
\hline $1 \mathrm{AA}$ & 09 & 16 & 0.707 & 5.657 & 17.678 & 0.0000 & 0.0000 & 0.0000 \\
\hline $1 \mathrm{AA}$ & 010 & 17 & 0.000 & 5.657 & 18.385 & 0.0000 & 0.0000 & 0.0000 \\
\hline $1 \mathrm{AA}$ & F1 & 18 & 19.092 & 4.950 & 18.385 & 0.0000 & 0.0000 & 0.0000 \\
\hline $1 \mathrm{AA}$ & $\mathrm{F} 2$ & 19 & 18.385 & 5.657 & 18.385 & 0.0000 & 0.0000 & 0.0000 \\
\hline $1 A A$ & F3 & 20 & 17.678 & 6.364 & 18.385 & 0.0000 & 0.0000 & 0.0000 \\
\hline $1 \mathrm{AA}$ & F 4 & 21 & 18.385 & 6.364 & 19.092 & 0.0000 & 0.0000 & 0.0000 \\
\hline $1 \mathrm{AA}$ & F5 & 22 & 17.678 & 5.657 & 19.092 & 0.0000 & 0.0000 & 0.0000 \\
\hline $1 \mathrm{AA}$ & $\mathrm{F} 6$ & 23 & 16.971 & 6.364 & 19.092 & 0.0000 & 0.0000 & 0.0000 \\
\hline $1 \mathrm{AA}$ & F7 & 24 & 16.263 & 6.364 & 18.385 & 0.0000 & 0.0000 & 0.0000 \\
\hline $1 \mathrm{AA}$ & F 8 & 25 & 15.556 & 6.364 & 17.678 & 0.0000 & 0.0000 & 0.0000 \\
\hline $1 \mathrm{AA}$ & F9 & 26 & 15.556 & 7.071 & 16.971 & 0.0000 & 0.0000 & 0.0000 \\
\hline $1 \mathrm{AA}$ & F10 & 27 & 16.263 & 6.364 & 16.971 & 0.0000 & 0.0000 & 0.0000 \\
\hline $1 \mathrm{AA}$ & F11 & 28 & 15.556 & 6.364 & 16.263 & 0.0000 & 0.0000 & 0.0000 \\
\hline $1 \mathrm{AA}$ & F 12 & 29 & 16.263 & 6.364 & 15.556 & 0.0000 & 0.0000 & 0.0000 \\
\hline $1 \mathrm{AA}$ & F13 & 30 & 16.971 & 5.657 & 15.556 & 0.0000 & 0.0000 & 0.0000 \\
\hline $1 \mathrm{AA}$ & F14 & 31 & 16.971 & 6.364 & 14.849 & 0.0000 & 0.0000 & 0.0000 \\
\hline $1 \mathrm{AA}$ & F15 & 32 & 16.971 & 5.657 & 14.142 & 0.0000 & 0.0000 & 0.0000 \\
\hline $1 \mathrm{AA}$ & F16 & 33 & 17.678 & 5.657 & 13.435 & 0.0000 & 0.0000 & 0.0000 \\
\hline $1 \mathrm{AA}$ & F17 & 34 & 18.385 & 6.364 & 13.435 & 0.0000 & 0.0000 & 0.0000 \\
\hline $1 \mathrm{AA}$ & F18 & 35 & 19.092 & 7.071 & 13.435 & 0.0000 & 0.0000 & 0.0000 \\
\hline $1 \mathrm{AA}$ & F19 & 36 & 18.385 & 7.071 & 14.142 & 0.0000 & 0.0000 & 0.0000 \\
\hline $1 \mathrm{AA}$ & F20 & 37 & 17.678 & 7.071 & 14.849 & 0.0000 & 0.0000 & 0.0000 \\
\hline $1 \mathrm{AA}$ & F 21 & 38 & 18.385 & 7.778 & 14.849 & 0.0000 & 0.0000 & 0.0000 \\
\hline $1 \mathrm{AA}$ & F 22 & 39 & 17.678 & 7.778 & 14.142 & 0.0000 & 0.0000 & 0.0000 \\
\hline $1 \mathrm{AA}$ & F23 & 40 & 17.678 & 8.485 & 14.849 & 0.0000 & 0.0000 & 0.0000 \\
\hline $1 \mathrm{AA}$ & F24 & 41 & 16.971 & 8.485 & 15.556 & 0.0000 & 0.0000 & 0.0000 \\
\hline $1 \mathrm{AA}$ & F25 & 42 & 16.263 & 8.485 & 14.849 & 0.0000 & 0.0000 & 0.0000 \\
\hline $1 \mathrm{AA}$ & F 26 & 43 & 16.971 & 9.192 & 14.849 & 0.0000 & 0.0000 & 0.0000 \\
\hline $1 \mathrm{AA}$ & F27 & 44 & 16.263 & 9.192 & 15.556 & 0.0000 & 0.0000 & 0.0000 \\
\hline $1 \mathrm{AA}$ & F 28 & 45 & 16.971 & 9.192 & 16.263 & 0.0000 & 0.0000 & 0.0000 \\
\hline $1 \mathrm{AA}$ & F29 & 46 & 16.263 & 8.485 & 16.263 & 0.0000 & 0.0000 & 0.0000 \\
\hline $1 \mathrm{AA}$ & F30 & 47 & 15.556 & 9.192 & 16.263 & 0.0000 & 0.0000 & 0.0000 \\
\hline $1 \mathrm{AA}$ & F31 & 48 & 14.849 & 9.899 & 16.263 & 0.0000 & 0.0000 & 0.0000 \\
\hline $1 \mathrm{AA}$ & F32 & 49 & 14.849 & 9.192 & 15.556 & 0.0000 & 0.0000 & 0.0000 \\
\hline $1 \mathrm{AA}$ & F33 & 50 & 14.849 & 8.485 & 16.263 & 0.0000 & 0.0000 & 0.0000 \\
\hline $1 \mathrm{AA}$ & F34 & 51 & 14.142 & 7.778 & 16.263 & 0.0000 & 0.0000 & 0.0000 \\
\hline $1 \mathrm{AA}$ & F35 & 52 & 14.849 & 7.778 & 15.556 & 0.0000 & 0.0000 & 0.0000 \\
\hline $1 \mathrm{AA}$ & F36 & 53 & 14.849 & 7.071 & 16.263 & 0.0000 & 0.0000 & 0.0000 \\
\hline
\end{tabular}




\begin{tabular}{|c|c|c|c|c|c|c|c|c|}
\hline $1 \mathrm{AA}$ & F37 & 54 & 14.142 & 6.364 & 16.263 & 0.0000 & 0.0000 & 0.0000 \\
\hline $1 \mathrm{AA}$ & F38 & 55 & 14.849 & 5.657 & 16.263 & 0.0000 & 0.0000 & 0.0000 \\
\hline $1 A A$ & F39 & 56 & 15.556 & 5.657 & 15.556 & 0.0000 & 0.0000 & 0.0000 \\
\hline $2 A B$ & 011 & 57 & 4.950 & 12.728 & 19.092 & 0.0000 & 0.0000 & 0.0000 \\
\hline $2 A B$ & 012 & 58 & 5.657 & 12.728 & 18.385 & 0.0000 & 0.0000 & 0.0000 \\
\hline $647 \mathrm{ZV}$ & H5 88 & 3892 & 16.263 & 19.092 & 16.971 & 0.0000 & 0.0000 & 0.0000 \\
\hline $648 \mathrm{ZW}$ & H589 & 3893 & 16.263 & 1.414 & 17.678 & 0.0000 & 0.0000 & 0.0000 \\
\hline $6497 X$ & H590 & 3894 & 19.092 & 1.414 & 17.678 & 0.0000 & 0.0000 & 0.0000 \\
\hline 19.79899 & 19.7 & 9899 & 19.79899 & & & & & \\
\hline
\end{tabular}

\section{References}

[1] L. Leibler, Theory of Microphase Separation in Block Copolymers, Macromolecules 13(6), 1602 (1980).

[2] A.E. Likhtman and A.N. Semenov, Stability of the OBDD structure for diblock copolymer melts in the strong segregation limit, Macromolecules 27(11), 3103 (1994).

[3] M.W. Matsen and M. Schick, Stable and unstable phases of a diblock copolymer melt, Phys. Rev. Lett. 72(16), 2660 (1994).

[4] M.W. Matsen and F.S. Bates, Block copolymer microstructures in the intermediate-segregation regime, J. Chem. Phys. 106, 2436 (1997).

[5] M. Takenaka, T. Wakada, S. Akasaka, S. Nishitsuji, K. Saijo, H. Shimizu, M.I. Kim, and H. Hasegawa, Orthorhombic Fddd Network in Diblock Copolymer Melts, Macromolecules 40(13), 4399 (2007).

[6] B. Miao and R.A. Wickham, Fluctuation effects and the stability of the Fddd network phase in diblock copolymer melts, J. Chem. Phys. 128, 054902 (2008).

[7] M.W. Matsen, The standard Gaussian model for block copolymer melts, J. Phys.: Condens. Matter 14(2), R21 (2002).

[8] K.A. Mauritz and R.B. Moore, State of Understanding of Nafion, Chem. Rev. 104, 4535 (2004).

[9] Michael Hickner, Hossein Ghassemi, Yu Seung Kim, Brian R Einsla, and James E McGrath, Alternative polymer systems for proton exchange membranes (PEMs), Chemical Reviews 104(10), 4587 (2004).

[10] M.J. Park and N.P. Balsara, Phase Behavior of Symmetric Sulfonated Block Copolymers, Macromolecules 41, 3678 (2008).

[11] Xin Wang, Sergey Yakovlev, Keith M. Beers, Moon J. Park, Scott a. Mullin, Kenneth H. Downing, and Nitash P. Balsara, On the Origin of Slow Changes in Ionic Conductivity of Model Block Copolymer Electrolyte Membranes in Contact with Humid Air, Macromolecules 43(12), 5306 (2010).

[12] Y. Rabin and J.F. Marko, Microphase separation in charged diblock copolymers: the weak segregation limit, Macromolecules 24(8), 2134 (1991).

[13] R. Kumar and M. Muthukumar, Microphase separation in polyelectrolytic diblock copolymer melt: weak segregation limit, J. Chem. Phys. 126, 214902 (2007).

[14] I. Nakamura, I., N.P. Balsara, and Z.-G. Wang, Thermodynamics of Ion-Containing Polymer Blends and Block Copolymers, Phys. Rev. Lett. 107, 198301 (2011).

[15] I. Nakamura, I. and Z.-G. Wang, Salt-doped block copolymers: ion distribution, domain spacing and effective [small chi] parameter, Soft Matter 8, 9356 (2012).
[16] P. Knychała, M. Banaszak, Park M. J., and N.P. Balsara, Microphase Separation in Sulfonated Block Copolymers Studied by Monte Carlo Simulations, Macromolecules 42(22), 8925 (2009).

[17] P. Knychała, M. Dzięcielski, M. Banaszak, and N.P. Balsara, Phase Behavior of Ionic Block Copolymers Studied by a Minimal Lattice Model with Short-Range Interactions, Macromolecules 46(14), 5724 (2013).

[18] P. Knychała, M. Banaszak, and N.P. Balsara, Effects of Composition on the Phase Behavior of Ion-Containing Block Copolymers Studied by a Minimal Lattice Model, Macromolecules 47(7), 2529 (2014).

[19] P. Knychała and M. Banaszak, Simulations on a swollen gyroid nanostructure in thin films relevant to systems of ionic block copolymers, European Physical Journal E 37, 67 (2014).

[20] J.E. Lennard-Jones, In On the Determination of Molecular Fields, volume 106, page 463 The Royal Society, 1924.

[21] S. Blonski, W. Brostow, and J. Kubat, Molecular-Dynamics Simulations of Stress-Relaxation in Metals and Polymers, Physical Review B 49, 6494 (1994).

[22] K. Kremer and G.S. Grest, ynamics of Entangled Linear Polymer Melts: A Molecular-Dynamics Simulation, J. Chem. Phys. 92, 5057 (1990).

[23] B. Hess, H. Bekker, H.J.C. Berendsen, and J.G.E.M Fraaije, LINCS: A Linear Constraint Solver for Molecular Simulations, Journal of Computational Chemistry 18, 1463 (1997).

[24] J.P. Ryckaert, G. Ciccotti, and H.J.C. Berendsen, Numerical Integration of the Cartesian Equations of Motion of a System with Constraints: Molecular Dynamics of n-Alkanes, Journal of Computational Physics 23, 327 (1977).

[25] T. Darden, D. York, and L. Pedersen, Particle Mesh Ewald: An $N$ - $\log (N)$ Method for Ewald Sums in Large Systems, J. Chem. Phys. 98, 10089 (1993).

[26] P. Ewald, Die Berechnung optischer und elektrostatischer Gitterpotentiale, Ann. Phys. 369, 253 (1921).

[27] M.P. Allen and D. Tildesley, Computer Simulation of Liquids, Clarendon Press Oxford, 1989.

[28] J. Kolafa and J.W. Perram, Cutoff Errors in the Ewald Summation Formulae for Point Charge Systems, Molecular Simulation 9, 351 (1992).

[29] S. Nose, A Unified Formulation of the Constant Temperature Molecular-Dynamics Methods, J. Chem. Phys. 81, 511 (1984).

[30] W.G. Hoover, Canonical Dynamics: Equilibrium PhaseSpace Distribution, Phys. Rev. A 31, 1695 (1984).

[31] W.F. Van Gunsteren and H.J.C. Berendsen, A Leap-frog Algorithm for Stochastic Dynamics, Molecular Simulation 1, 173 (1988). 
[32] L. Verlet, Computer Experiments on Classical Fluids. I. Thermodynamical Properties of Lennard-Jones Molecules, Physical Review 159, 98 (1967).

[33] Gromacs User Manual 4.0, ftp://ftp.gromacs.org/pub/manual/ manual-4.0.pdf, Available: 30.08.2015.

[34] T.M. Beardsley and M.W. Matsen, Monte Carlo phase diagram for diblock copolymer melts, Eur. Phys. J. E 32(3), 155 (2010).
[35] D. Van Der Spoel, E. Lindahl, B. Hess, G. Groenhof, A.E. Mark, and H.J.C. Berendsen, GROMACS: Fast, Flexible, and Free, J. Comput. Chem. 26, 1701 (2005).

[36] Tabulated Potentials, http://www.gromacs.org/Documenta tion/How-tos/Tabulated_Potentials, Available: 30.08.2015.

[37] User Specified non-bonded potentials in gromacs, http://www. gromacs.org/@api/deki/files/94/=gromacs_nb.pdf, Available: 29.10.2016.

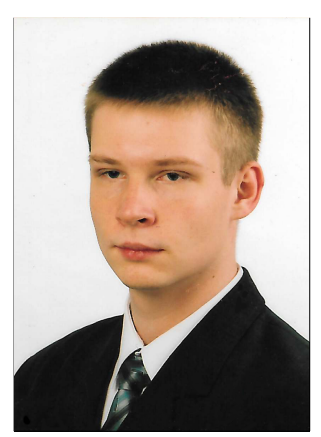

Michał Dzięcielski

received his PhD degree in Physics in 2016 from the Adam Mickiewicz University in Poznań. Since 2016 he has been working at the Institute of Socio-Economic Geography and Spatial Management of the Adam Mickiewicz University in Poznań. His main areas of interest are: computer simulations, complex systems, self-organization and polymer physics.
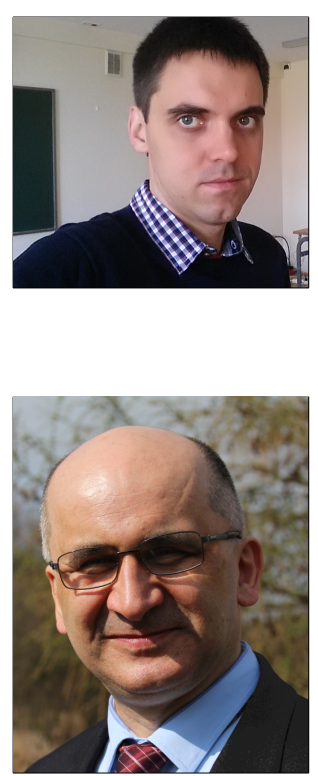

Piotr Knychała graduated in Computer Science in 2007 from the Adam Mickiewicz University in Poznań, received his PhD degree in Physics in 2012 from the Adam Mickiewicz University in Poznań. Since 2011 he has been working at the Faculty of Polytechnic of the President Stanislaw Wojciechowski University School of Applied Sciences in Kalisz. His main research interests include polymer melts and solutions, block copolymer nanostructures, ionic copolymers, and coarse-grained models of biopolymers investigated by the computer simulations.

Michał Banaszak graduated in Theoretical Physics in 1985 from the Adam Mickiewicz University in Poznań. He received his PhD degree in Physics in 1991 from Memorial University in St. John's, Canada, specializing in Polymer Physics. From 1992 to 1995 he worked as postdoctoral fellow in Exxon Research \& Engineering Co. in Annandale, New Jersey, USA, working on theory and modeling of polymer solutions. From 1995 to 1997 he worked in the Chemistry Department of UMIST in Manchester, UK, as a research associate, specializing in large-scale computer simulations of ionic copolymers. In 1997 he joined the Adam Mickiewicz University, obtaining DSc degree (habilitation) in 2004 in Physics (specialty: Soft Matter Physics and Computer Simulations), and later professorship in Physics. His main interest is in developing new models and theories for nanoscale self-assembly of various polymer systems. He also collaborates with experimentalists, using large-scale computing. 